\title{
Insights on the left ventricular thrombus in patients with ischemic dilated cardiomyopathy
}

\author{
Elsayed M. Mehana ${ }^{{ }^{*}} \mathbb{D}$, Abeer M. Shawky ${ }^{2}$ and Heba S. Abdelrahman ${ }^{3}$
}

\begin{abstract}
Background: Left ventricular thrombus (LVT) formation represents a common complication of dilated cardiomyopathy (DCM). LVT is usually underestimated with transthoracic echocardiography (TTE) while cardiac magnetic resonance imaging (CMRI) is promising as an alternative imaging modality for cardiac thrombus detection. The study aims to compare TTE and CMRI in their ability to detect LVT, also, to assess the clinical and imaging parameters to determine variables that may predispose for thrombus formation. The study population includes seventy-six patients with ischemic DCM. They were divided into 2 groups based on the presence of LVT as detected by delayed-enhancement CMRI (DE-CMRI) [Group A included 20 patients with a LVT and Group B included 56 patients without].

Results: All of the current study population had ischemic DCM with left ventricular ejection fraction (LVEF) $<50 \%$. DECMR detected thrombus in 20 cases of the studied population that represented group A. From group A, conventional TTE detected LVT only in 8 and cine-CMR detected 13 cases out of the out of 20 cases. The ejection fraction of the left ventricle as measured by functional CMRI was significantly lower in group A $(P=0.045)$. Interestingly, the myocardial scarring in group A was seen significantly more extensive than in group $B$ (the $P$ value is $<0.00001$ ), paralleling the increased prevalence of thrombus.
\end{abstract}

Conclusions: DE-CMRI provides superiority for the detection of LVT compared with standard TTE or cine-CMRI and the amount of myocardial scarring detected by DE-CMRI can be considered an independent marker for thrombus presence.

Keywords: Left ventricular thrombus, Dilated cardiomyopathy, Transthoracic echocardiography, Cardiac magnetic resonance imaging

\section{Background}

Patients with dilated cardiomyopathy (DCM) are at increased short and long term risks for thromboembolic events due to left ventricular thrombus (LVT) formation. Thrombosis in the cardiac cavity is associated with a reduction of the left ventricular ejection fraction $(E F)$ and related to kinetic disorders of the infarcted and

*Correspondence: sayedmehana9@gmail.com; elsayed.mehana@alexu.edu. eg

${ }^{1}$ Department of Diagnostic Radiology, Medical Research Institute, Alexandria University, Alexandria, Egypt

Full list of author information is available at the end of the article peri-infarction areas. Early detection of LVT allows for the early initiation of anticoagulation therapy to reduce the likelihood of embolization [1]. Transthoracic echocardiography (TTE) is the first choice diagnostic tool in such patients. However, thrombus formation is usually underestimated with echocardiography because it is difficult to distinguish normal myocardium from thin mural thrombi [2].

One of the diagnostic tools that provide high contrast resolution images of the anatomy of the LVT is cardiovascular magnetic resonance imaging (CMRI). Cine-CMRI (CMRI without a contrast agent such as gadolinium) seems to be less suitable for LVT detection 
[3]. Delayed-enhancement CMRI (DE-CMRI) using gadolinium contrast has been well validated as a mean of characterizing viable and infarcted myocardium based on contrast uptake patterns [4]. DE-CMR differentiates thrombus from the surrounding myocardium based on tissue characteristics as thrombus is avascular and thus characterized by an absence of contrast uptake [5].

The current study aimed to zoom on LVT in patients with ischemic DCM from many aspects. Firstly, by determining the clinical and imaging predictors for LVT formation in patients with DCM. Secondly, by assessing the ability of conventional echo and cine-CMRI in the detection of thrombus compared to the gold standard DE-CMRI.

\section{Materials and methods Study population}

This study is a retrospective, single-centre observational study. The study population consisted of 76 consecutive patients with systolic dysfunction of a clinical outpatient facility of a private hospital, between July 2018 and July 2020. Those patients were referred to the CMRI unit for evaluation of myocardial viability. Medical history was taken to assess potential predictors of the presence of LVT; including cardiac risk factors, medication regimen, and information regarding prior myocardial infarction. We included only patients with ischemic dilated cardiomyopathy. We excluded patients with dilated cardiomyopathy due to causes other than ischemia, those with contraindications for gadolinium administration or with uncompleted clinical records. The studied patients were divided, according to the presence or absence of thrombi as detected by DE-CMRI, into two groups of patients (Group A with a left ventricular thrombus and Group B without). The comparison was made between the two groups regarding age, gender, presence of mitral regurgitation, left ventricular ejection fraction, and apical wall motion abnormality. Using DE-CMRI as a reference standard, a comparison was made between standard TTE and cine-CMRI regarding their ability to detect LVT in group A.

\section{Two-dimensional and doppler echocardiography}

Echocardiographic examinations were performed using a Phillips machine with standard views and techniques. Two-dimensional (2D) images were obtained with the use of a $3.5 \mathrm{MHz}$ transducer. Variable gain and depth settings were used to optimize epicardial, endocardial and thrombus margin definition. The images were obtained with the patient in the left lateral position at the end-expiration.

The echocardiographic definition of a thrombus was a consistent mass of refractile echoes that had well-defined margins adjacent to an area of the LV wall which is hypokinetic or akinetic, projected into the left ventricular cavity, easily distinguishable from the endocardium and the other intra-cardiac structures such as anomalous left ventricular chords or trabeculations, and seen in more than one view and visible throughout the systole and diastole [6]. The maximal thrombus size was measured perpendicular to the myocardium from the endocardial border extending to the innermost border of the thrombus-blood interface when viewed using apical views.

Left ventricular dimensions were determined by the leading-edge to edge method and the determination of left ventricular ejection fraction (LVEF) was calculated by using the biplane modified Simpson's method. Dilated cardiomyopathy was diagnosed, in the presence of $\mathrm{LVEF}<50 \%$ and LV end-diastolic diameter (LVEDd) of $>56 \mathrm{~mm}$ [7].

Wall motion abnormality at the apex (which was the site of thrombus in all of our cases), as determined by 2D imaging, was defined as a lack of wall motion (akinesia) or a paradoxical expansion (dyskinesia) observed during systole (Fig. 1) [8].

Mitral regurgitation, as determined by Doppler colour flow imaging, was semi-quantitative by relating the area of the regurgitant jet to the left atrial area, with a ratio of $<20 \%$ being mild mitral regurgitation (Grade 1), 20 to $40 \%$ being moderate mitral regurgitation (Grade 2) and a ratio of $>40 \%$ being severe mitral regurgitation (Grade 3) [9].

\section{Cardiac magnetic resonance imaging}

All CMR examinations were performed on a 1.5-T MRI system (Avanto, Siemens Healthcare, Erlangen, Germany) equipped with a 32-element cardiac coil array. All scans were ECG-gated for synchronization with the cardiac cycle and performed in end-expiration and following a local standard protocol that included the following sequences:

1. Three localizing single-shot steady-state sequences in the three orthogonal planes, followed by axial, sagittal, and coronal multi-slice half-Fourier-acquired single-shot turbo spin-echo (HASTE).

2. Cine sequences: Steady-state free precession (SSFP)oriented 2-chamber vertical long-axis, 4-chamber horizontal long-axis, 3-chamber, and short-axis views for studying the kinetics of the right and left ventricles. Acquisition time, 7-12 s each slice; matrix, $192 \times 192$; flip angle, $180^{\circ}$; echo time (ET), $1.69 \mathrm{~ms}$.

3. Phase-contrast sequence to quantify the amount and severity of the mitral regurgitation in suspected cases. This sequence was planned with one slice perpendicular to the mitral valve using four chambers and two-chamber views, velocity encoding 

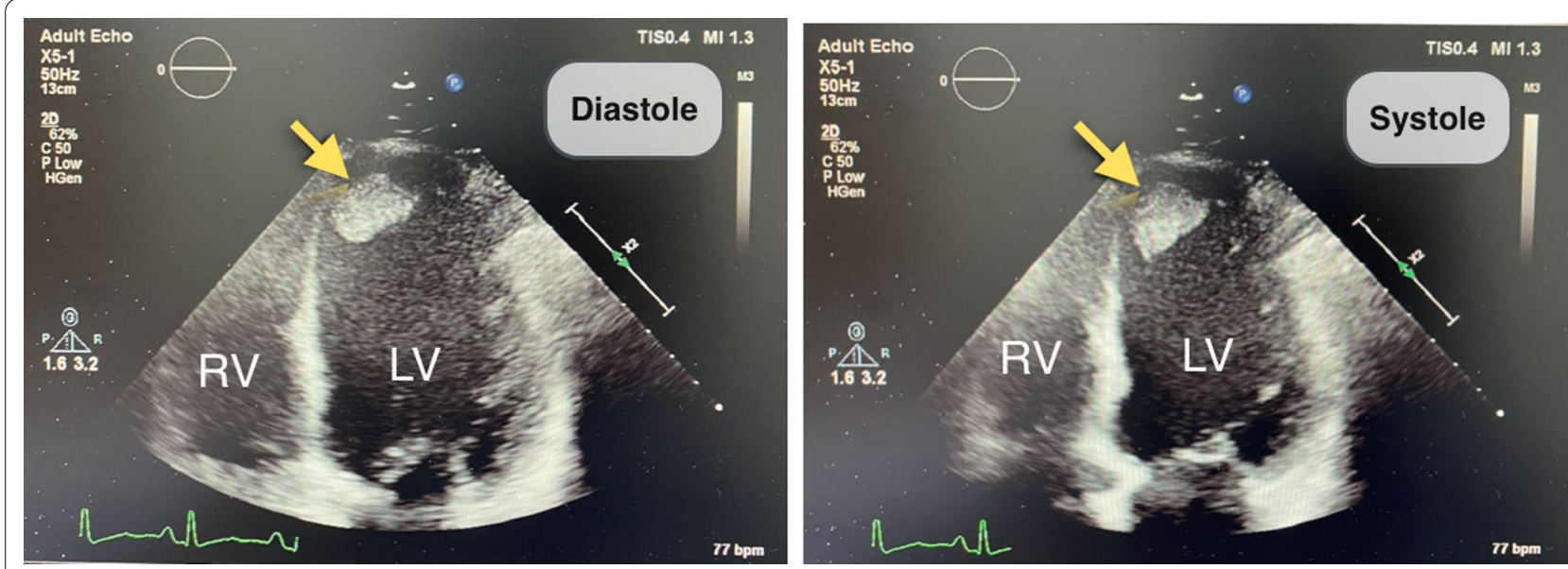

Fig. 1 Apical four-chamber transthoracic echocardiographic appearance of a mobile, protruding thrombus (yellow arrows; in diastole and systole) in the left ventricular apex in a patient with dilated cardiomyopathy with apical akinesia. This thrombus appears more echo dense than the surrounding myocardium. LV left ventricle, LA left atrium

$(\mathrm{VENC})=150 \mathrm{~cm} / \mathrm{s}$ for normal flow, retrospective gating, and short TE for optimal flow sensitivity.

4. Phase-sensitive inversion recovery (PSIR) sequences in free-breathing for studying early gadolinium enhancement (EGE) 1-3 min after intravenous administration of gadolinium $(0.1-0.2 \mathrm{mmol} / \mathrm{kg})$ with longer inversion times than those for LGE around (450-500 ms) for diagnosis of intracavitary thrombi, then late gadolinium enhancement (LGE) performed 10-15 min to detect myocardial infarction/ scarring; inversion time (300-400 ms). FOV, $244 \times 300 \mathrm{~mm}^{2}$; matrix, $156 \times 256$.

Quantitative evaluation for LVEF was carried out using Siemens imaging software Syngo. Systolic dysfunction was defined as a LVEF below 56\% measured quantitatively on short-axis cine-CMR [10]. The presence and transmural extent of the enhancement were reported and the number of enhancing segments was counted.

\section{Statistical methods}

Normally distributed continuous data were expressed as mean $\pm S D$ and between-group comparisons were performed using 2-sample t-tests. Chi-square tests were used to compare discrete data between groups; in those cases, in which the expected cell count was $<5$, the Fisher exact test was used. We analyzed the value of clinical variables for predicting the presence of thrombus by DE-CMR. All clinical variables were considered and the incremental value of adding LVEF by cine-CMR and myocardial scarring by DE-CMR was assessed using likelihood ratio tests. All statistical tests were 2-tailed; values of $\mathrm{p}<0.05$ were regarded as significant.

\section{Results}

The study population was composed of 76 patients with ischemic DCM. The mean age of the studied patients was $54.1 \pm 9.5$ years. Male patients represented $86.8 \%$ of all the studied patients. The patients' population were divided into two groups based on the presence of LVT; Group A with thrombus $(n=20[26.3 \%])$ and Group B without thrombus $(n=56[73.7 \%])$.

Clinical characteristics between the 2 groups of patients did not differ concerning the age, gender, and risk factors for coronary artery disease (Table 1). There was also no statistically significant difference between the two groups regarding the prevalence of functional mitral regurgitation (MR) which was noted by TTE in 8 patients out of $20(40 \%)$ in group A and 34 patients out of $56(44.7 \%)$ in group B.

The apex of the left ventricle was involved by the thrombus in all of the studied patients. All studied patients had akinetic or dyskinetic apex that was evaluated by TTE and Cine-CMRI and showed no statistically significant difference between group A and group $B$.

Upon comparing the two groups of patients concerning their LVEF as determined by Cine-CMR and TTE, there was evidence of advanced systolic dysfunction in both groups. The mean difference between the two groups was $6.2 \%$ using both Cine-CMRI and echocardiography; which is statically significant $(\mathrm{P}=0.03$ and 0.002 ; for both techniques respectively) (Table 2).

Interestingly, the myocardial scarring as detected by DE-CMRI, in the form of transmural enhancement or sub-endocardial enhancement more than $50 \%[11,12]$, in group A was seen significantly more extensive than in group $B$ according to the number of left ventricular 
Table 1 : Baseline clinical characteristics of the whole study group and patients with and without LV Thrombus (Group A and B; respectively)

\begin{tabular}{|c|c|c|c|c|}
\hline Variables & All patients $(n=76)$ & Group A $(n=20)$ & Group B $(n=56)$ & $P$ value \\
\hline Mean age (years) & $53 \pm 11$ & 49 & 55 & 0.2 \\
\hline Male (sex) & $66(86.8 \%)$ & 18 & 48 & 1.0 \\
\hline \multicolumn{5}{|c|}{ Coronary disease risk factors } \\
\hline Hypertension & 32 & 6 & 26 & 0.3 \\
\hline Diabetes mellitus & 35 & 8 & 27 & 0.54 \\
\hline Dyslipidemia & 26 & 7 & 19 & 1.0 \\
\hline Smoker & 39 & 13 & 26 & 0.2 \\
\hline
\end{tabular}

$n=$ Number of patients; Group A = Patients with dilated cardiomyopathy and left ventricular apical thrombus; Group $2=$ comprised of patients showing no thrombus; $P$ value is significant if 0.05 or less using T-test for age difference and Easy Fisher Exact test for the rest

Table 2 Comparison of left ventricular ejection fraction between group $A$ and group $B$

\begin{tabular}{llllll}
\hline & \multicolumn{2}{l}{ Cine-CMRI } & & & ECHO \\
\cline { 2 - 3 } \cline { 5 - 6 } & Group A & Group B & & Group A & Group B \\
\hline Numbers of patients $(n)$ & 20 & 56 & & 20 & 56 \\
Mean EF \% & $26.2 \pm 11.5$ & $32.4 \pm 10.4$ & & $24 \pm 5.3$ & $30.2 \pm 6.8$ \\
Mean differences & 6.2 & & 6.2 & \\
$P$ value & 0.03 & & 0.002 & \\
\hline
\end{tabular}

$n=$ Number of patients; Group A = Patients with dilated cardiomyopathy and left ventricular apical thrombus; Group $2=$ comprised of patients showing no thrombus; $P$ value is significant if 0.05 or less using One-Way ANOVA test; $\mathrm{CMRI}=$ cardiac magnetic resonance imaging; $\mathrm{ECHO}=$ trans-thoracic echocardiography; Data are shown as mean \pm standard deviation or number (\%); $\mathrm{EF}=$ Ejection Fraction

segments affected (The $t$ value was 6.9 and the $P$ value was $<0.00001)$. The mean number of segments affected was higher in group A than in group B $(7.3 \pm 3.3$ and $4.2 \pm 2.1)$, paralleling the increased prevalence of thrombus.

Thrombi were detected by DE-CMRI in twenty cases that represented $26.3 \%$ of our studied population; out of them, only 8 cases $(40 \%)$ were detected by conventional echocardiography while 12 cases were missed (Fig. 2). TTE also detected LVT in 2 cases in group B which were considered as a false positive result. CineCMRI detected intracardiac thrombi in 13 out of these 20 cases with a prevalence of $65 \%$ which was not statistically better than conventional TTE $(\mathrm{P}=0.02)$. Comparing with DE-CMRI, detection of LVT in both TTE and cine-CMRI was significantly less $(\mathrm{P}=0$ and 0.008 ; respectively) (Fig. 3).

In the current study, all the thrombi were involving at least the ventricular apex, adherent to the wall with an average size of $1.9 \pm 0.14 \mathrm{~cm}$ as detected by DE-CMRI and of $2.5+1.14 \mathrm{~cm}$ as detected by conventional echocardiography (Fig. 4).

\section{Discussion}

Ventricular thrombus formation in DCM reflects the presence of factors that represent Virchow's triad in the ventricle including local myocardial injury, reduced wall motion, and slow flow of the blood [13]. The clinical importance of LVT is that it carries the risk of embolic events. The incidence of LVT in the current study was $28.3 \%$, which was higher than the incidence in other prospective studies $(0.7-8 \%)$ [14, 15]. This can be explained by that, in the current study, we made a zoom on LVT in patients with ischemic DCM who were suspected to have thrombus.

In the current study, there was no significant difference in clinical variables between the two groups of studied patients regarding hypertension, dyslipidemia, smoking and diabetes mellitus and these results matched with Jiang and others in 2015 [14].

In the current study, all patients were on beta-blockers. In the literature, there is a controversy regarding the negative influence of beta-blockers in the occurrence of LVT in patients with DCM. Many studies reported a higher frequency of thrombus development in those patients when treated with beta-blockers and could be explained by the negative inotropic action of these drugs and thus increased blood stasis that contributes as one of the three components of Virchow's triad facilities the formation of LVT. Turpie et al. in 1989; reported an increased occurrence of thrombus in patients with anterior myocardial infarction after oral b-blocker therapy [16]. Un matching results were described in the GISSI-2 study that observed the same rate of LVT in patients with or without atenolol [17].

Secondary mitral regurgitation (MR) is an important issue in DCM patients. It is not due to a disease of the leaflets but to the symmetrical or asymmetrical dilation of the left ventricle [18]. It was reported that MR prevents thrombus formation in patients with DCM and this protective effect can be explained by increasing early 

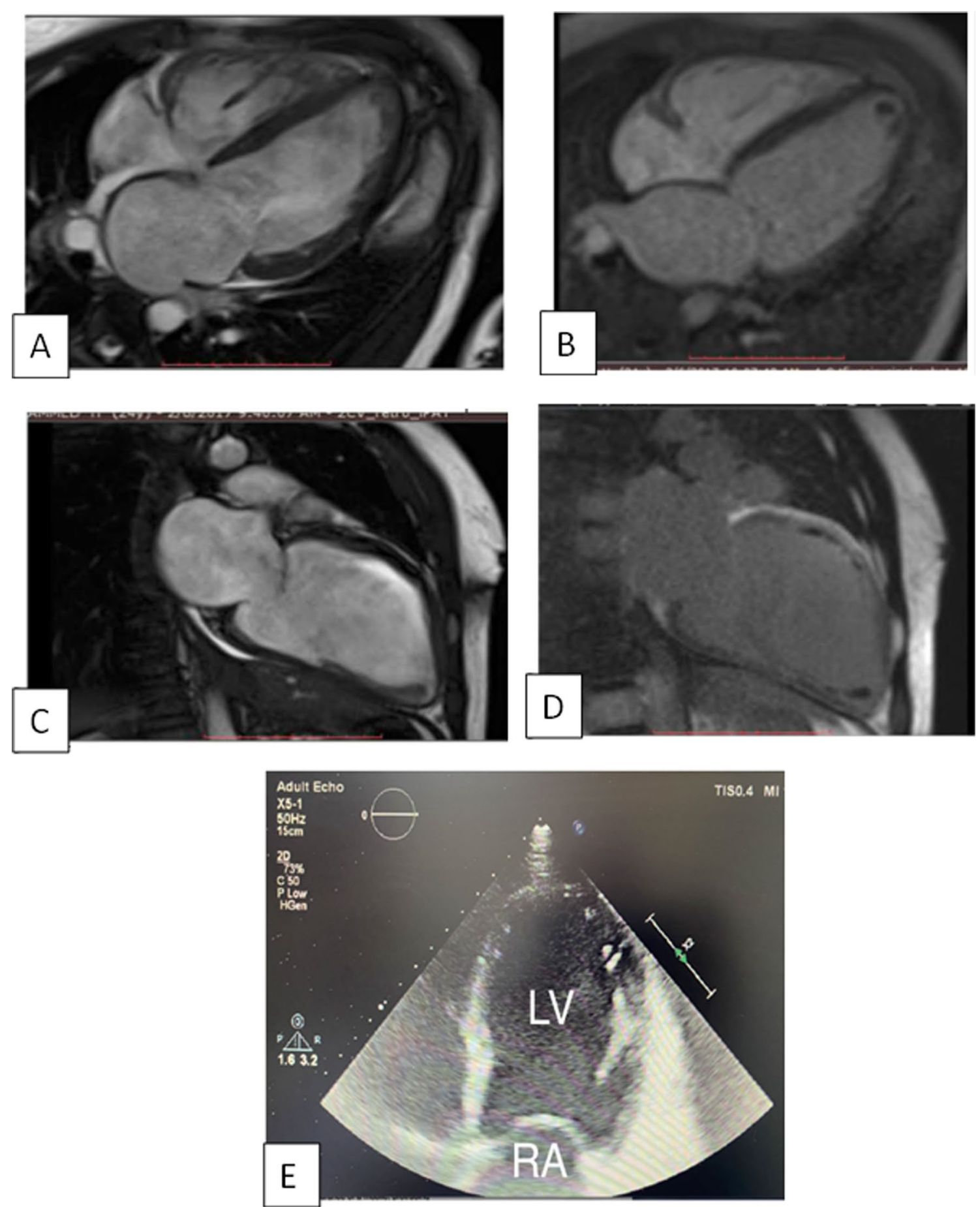

Fig. 2 Illustrative example of Comparing echocardiography and cine-CMRI by DE-CMRI in diagnosis of LVT. In this case, while the standard apical four-chamber Echocardiographic view (e) was inconclusive, cine-CMR imaging provided a better appreciation of the thrombus, four chamber view (a) and (Additional file 1: Movie 1) and two-chambers long-axis view (c) and (Additional file 2: Movie 2). Appearance of the same thrombus more appreciated using DE-CMRI four chamber view (b) and two-chamber long axis view (d) 

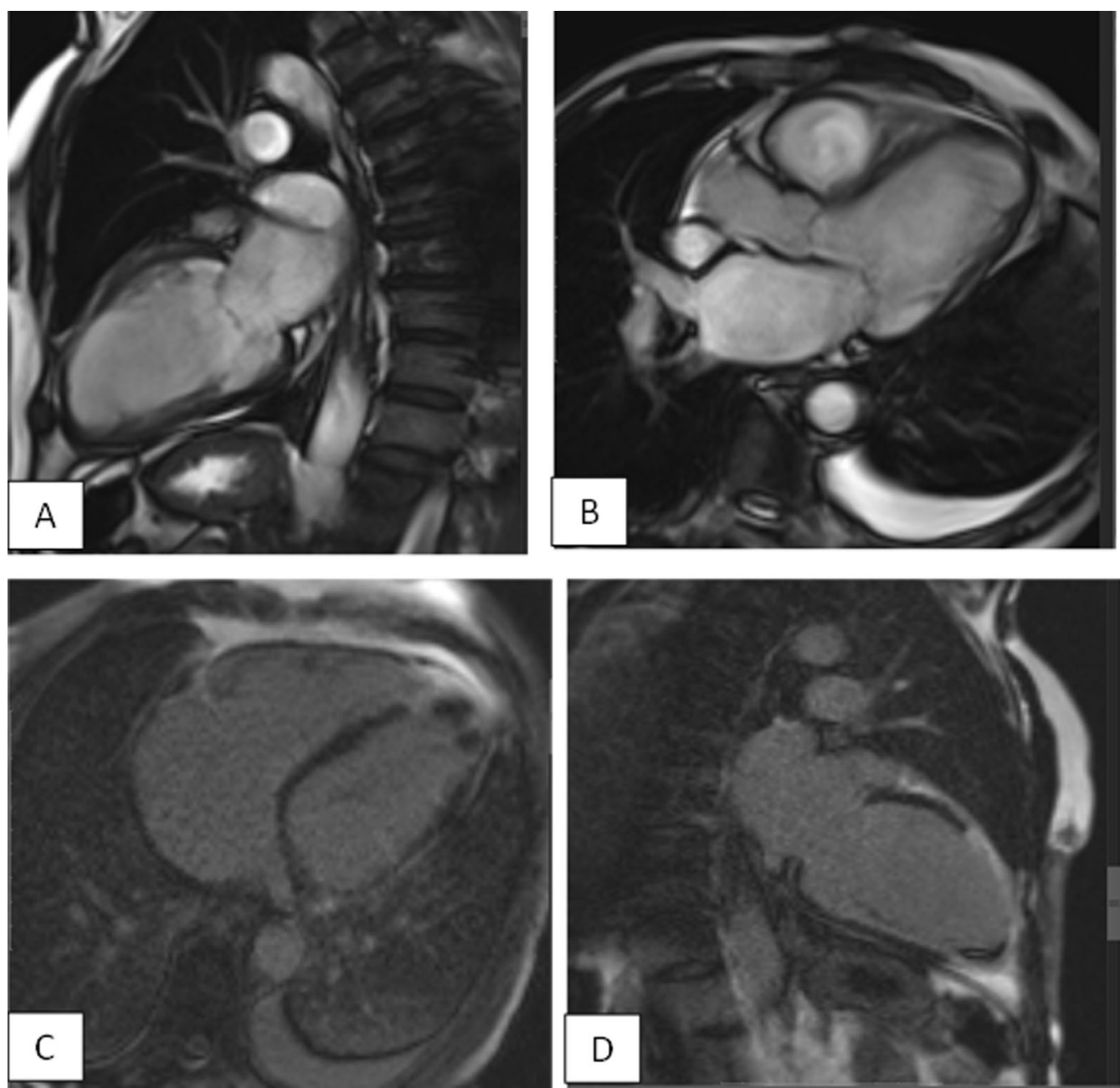

Fig. 3 69-year male with CAD. Apical dilatation is appreciated in cine 2 chambers long axis view (a) and (Additional file 3: Movie 3) and three-chambers view (b) and (Additional file 4: Movie 4) while the thrombus couldn't be visualized. Thrombus can be easily recognized in delayed post-contrast views on top of transmural enhancement of the underlying myocardium; four-chambers (c) and two-chambers long-axis (d)

diastolic flow velocities at the mitral annulus level and along the entire length of the LV, protecting the LV cavity from a slow blood flow pattern which is thrombogenic [19]. In the currently studied patients, no statistically significant difference was found between patients with or without LVT in presence of functional MR. MR was detected in $40 \%$ of group A and $44.7 \%$ of group B, which is consistent with the study of Garg et al. in 2019 [19], suggesting that this is not a major discriminatory variable in the formation of thrombus. However, Maze and others in 1989 noted a higher prevalence of MR in the no thrombus group [20]. Therefore, whether MR in patients with DCM is capable of decreasing blood stasis and LVT formation needs to be furtherly evaluated.

LVT are typically located in areas of reduced wall motion and adjacent to scars. The wall motion abnormality at the apex, which was the site of the thrombus in all cases in the current study, was not significantly different between groups A and B. All studied patients had akinetic or dyskinetic apex. The current study results were concordant with Maze et al., as they stated that no significant statistical difference between the thrombus and non-thrombus groups concerning the motion abnormality at the apex [20].

The presence of LVT is significantly related to the severity of LV functional impairment in patients with DCM [21]. Blood stagnation in the weak non-contractile segment of the ventricle plays a major role in the formation of thrombi [22]. In the current study, the two groups of patients showed evidence of advanced systolic dysfunction but more in group A with thrombi than in group B without. Several studies have shown that increased left ventricular internal diastolic diameters and low EF are independent predictors of LVT formation [6,23]. Timely 

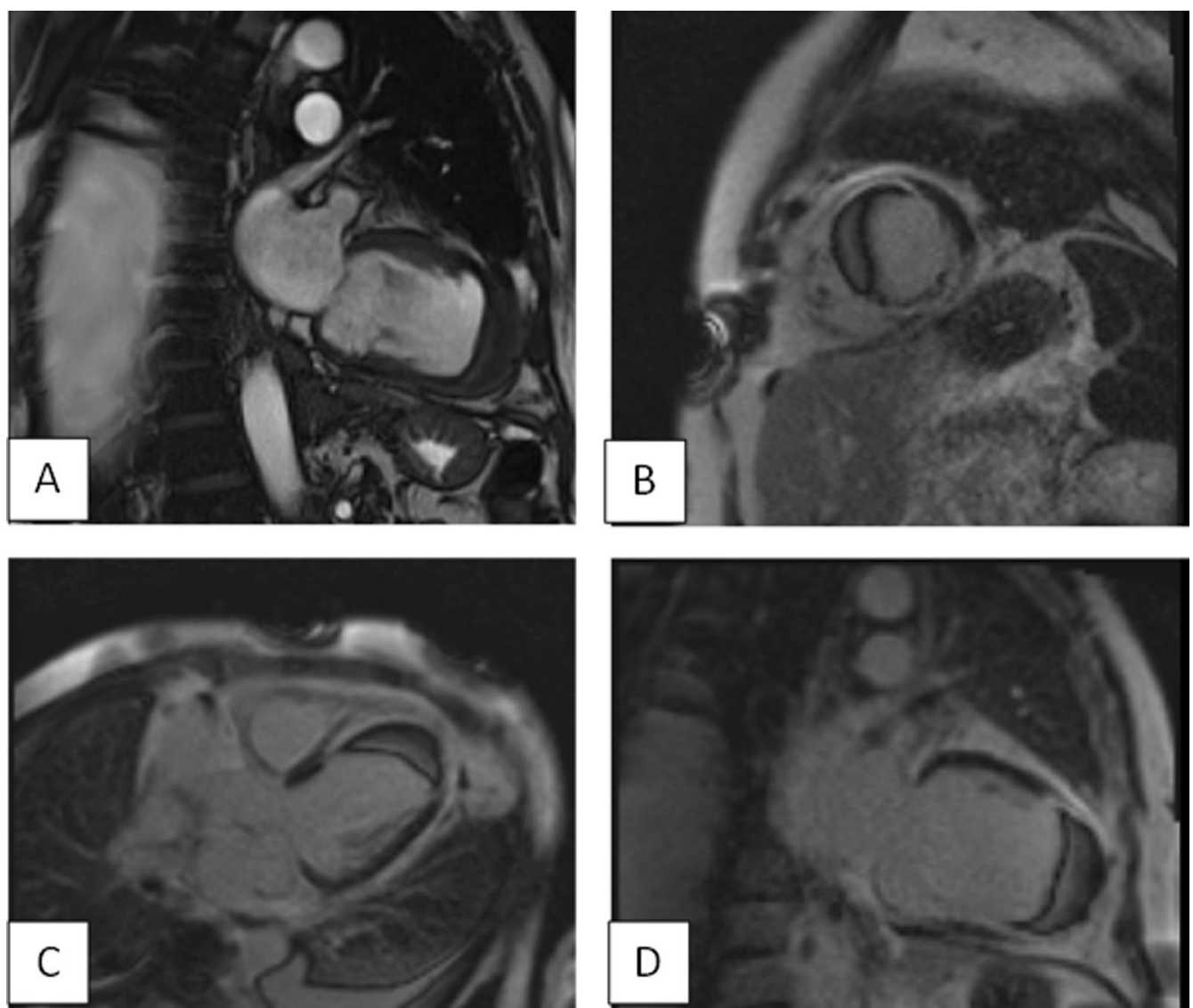

Fig. 4 a Long axis 2 chambers view (white blood) shows an isointense intraluminal lesion with underlying apical akinesia in cine sequence (Additional file 5: Movie 5). Delayed post contrast sequences in short axis (b), 4 chambers view (c) and 2 chambers long axis view (d) show nulled myocardial signal with transmural mid and apical septal enhancement reflecting non-viable myocardium and intraluminal thrombus

identification of LVT in patients with reduced EF is necessary to avoid delays in treatment with systemic anticoagulation. This identification is pretty important in patients with $\mathrm{LV}-\mathrm{EF} \leq 30 \%$ and sinus rhythm as prophylactic systemic anticoagulation is not supported in this group of patients relative to the increased risk of bleeding [24]. Therefore, advanced systolic dysfunction per se could not be used as an independent factor and a rationale for early treatment with prophylactic anticoagulant therapy to reduce the likelihood of embolization among the patients with ischemic DCM as the risk of bleeding also increased.

It has been noticed that the increase in the prevalence of LVT was paralleled by an increase in the scar burden. In the current study, the myocardial scarring as detected by DE-CMRI was seen significantly more extensive in patients with LVT than in patients without thrombus which matched with Kaolawanich and Boonyasirinant in 2019 as they stated that extensive apical scarring was found more significant in patients with thrombus than those without thrombus $(63.3 \%$ vs $23.3 \%$;
$P$ value $<0.0001)[25]$. Therefore, apical scarring can be considered as additional evidence demonstrating the significant value of apical changes and the amount of myocardial scarring as independent markers for thrombus formation.

In the current study, the thrombus prevalence was often higher among patients with extensive myocardial scarring. This might confirm the concept of "the independent value of myocardial scarring" that might in part explain the marked difference in thrombus prevalence regardless of the severity of systolic dysfunction. In 2008 Weinsaft and others identified a low LVEF and myocardial scarring as independent risk factors of LVT formation [26].

Echocardiography is a widely employed modality in the diagnosis of LVT because it is readily available, safe, relatively cheap, and convenient but it is operator-dependent. In a setting of adequate imaging of the heart, TTE provides excellent specificity (85-90\%) and sensitivity (95\%) in detecting LVT [24]. In the current study, standard TTE was the first-line imaging modality used to screen for 
LVT. However, it was a challenge because TTE detects thrombus based on anatomical characteristics thus having a high ability to detect large and protuberant thrombi but limited in small and mural thrombi.

CMRI for detection of LVT in DCM is potentially more advantageous, compared with standard TTE not only in providing better morphological definition (anatomical characteristics) by providing cine imaging but also can characterize and differentiate LVT from other structures after contrast administration [27]. DE-CMR differentiates thrombus from surrounding myocardium as thrombus is avascular and thus characterized by an absence of contrast uptake. On the other hand, cine-CMRI without a contrast agent may be relatively insensitive for thrombus detection because thrombus may be camouflaged with the surrounding myocardium [4].

From the studied patients, routine TTE failed to detect thrombus in 12 out of 20 cases (60\%). These results are consistent with Mollet et al. in 2002 who reported that among 12 patients with thrombus detected by CMRI, $58 \%$ (7 out of 12) were not detected by echocardiography [28]. Also, the current results matched the other studies that compared DE-CMRI and TTE and stated that DECMRI is superior for the detection of $\operatorname{LVT}[29,30]$. The limitations of TTE in detecting LVT were probably due to intra and inter-observer variation for detection of LVT that are lower for CMRI in comparison to TTE. One of the other factors that decreased detection of LVT in TTE is a poor acoustic window and it may be difficult to fully interrogate a very dilated ventricle if acoustic windows are limited. Broncano et al. in 2020 stated that cardiac MRI is not limited by acoustic windows as is TTE [31].

All of LVT in the current study patients involved the apical segments and it is well known that LVT is most frequently seen in the left ventricular apex as a common location in patients with ischemic as well as nonischemic DCM [32].

Taken together, DE-CMR is usually interpreted in conjunction with cine-CMR, therefore the current study cannot permit conclusions to be made regarding the utility of DE-CMR alone for LVT detection. We used DECMRI as a gold standard in the detection of LVT as it is better than standard echocardiography and cine-CMRI alone. In the current study, cine-CMRI detected LVT in 13 out of 20 cases detected by DE-CMRI, with a prevalence of $65 \%$ which was not statistically significant when compared with TTE in the detection of LVT in the same group. These results matched with Weinsaft and others in 2011 who stated that DE-CMRI was the most accurate modality for detection of LVT, followed by cine-CMRI, and finally standard TTE [33].

Finally, it is very important to avoid false-positive results occasioned by endocardial elastosis, trabeculae, false tendons near-field clutter, lipomatous metaplasia, and artefacts among others [7]. In the current study, DECMRI did not detect LVT in cases of Group B, however, LVT was detected by TTE in two cases of this group, which may be false-positive results.

The present study has some limitations. Firstly, it was a small single-centre study and further studies which include more patients or multicenter studies are necessary for the validation of our results. Secondly, being a retrospective study, the outcome of LVT in the studied patients could not be evaluated. Thirdly, assessment of other clinical risk factors associated with LVT in patients with DCM has not been done, making the assessment of causality rather inconclusive.

\section{Conclusions}

Multiple imaging modalities are required for detailed and comprehensive evaluations of patients with DCM and suspected LVT. TTE is the imaging modality of choice in those patients as it is cheap, more available and disadvantage of the contrast and possibility of nephrogenic systemic fibrosis, especially in repeated times [33]. However, our study showed that DE-CMRI added value towards competing TTE and Cine-CMRI modalities relies on its excellent tissue characterization of LVT with potentially relevant therapeutic and prognostic implications. DECMRI provided superiority for detection of the amount of myocardial scarring that can be considered as an independent marker for thrombus formation.

Developing strategies based on CMRI for the detection of cardiac thrombi and flow stasis is of great clinical interest. Therefore, further investigations and efforts are needed to improve the evaluation of slow flow and early thrombi detection in patients with DCM and to ascertain whether DE-CMRI findings can be used to guide anticoagulant therapy and improve clinical outcomes among those population of patients at risk for LVT and related complications.

\section{Abbreviations}

DCM: Dilated cardiomyopathy; LVT: Left ventricular thrombus/thrombi; EF: Ejection fraction; TTE: Transthoracic echocardiography; CMRI: Cardiovascular magnetic resonance imaging; DE-CMRI: Delayed-enhancement CMRI; 2D: Two-dimensional; HASTE: Half-Fourier-acquired single-shot turbo spin-echo; EGE: Early gadolinium enhancement,; MR: Mitral regurgitation.

\section{Supplementary Information}

The online version contains supplementary material available at https://doi. org/10.1186/s43055-021-00628-5.

Additional file 1. Movie 1. Cine Four-chamber view white blood sequence for the case demonstrated in figure (2) showing a left ventricular thrombus. 
Additional file 2. Movie 2. Cine Two-chambers long-axis view white blood sequence for the case demonstrated in figure (2) showing a left ventricular thrombus.

Additional file 3. Movie 3. Cine two chambers long-axis view for the case demonstrated in figure (3), the thrombus is not visualized.

Additional file 4. Movie 4. Cine four chambers view for the case demonstrated in figure (3), the thrombus is not visualized.

Additional file 5. Movie 5. Cine long-axis view for the case demonstrated in figure (4) showing apical akinesia with the thrombus is seen isointense to the myocardium.

\section{Acknowledgements}

Not applicable.

\section{Authors' contributions}

EM: contributed to study design, study selection, collection of data, statistical analyses, data interpretation, and writing the manuscript. AS: contributed to study design, collection of data, interpreted the data and writing the manuscript. HA: contributed to study selection, data interpretation, and provided vital reviews of the manuscript. All authors read and approved the final manuscript.

\section{Funding}

The author state that this work has not received any funding.

\section{Availability of data and materials}

The datasets used and/or analysed during the current study are available from the corresponding author on reasonable request.

\section{Declarations}

\section{Ethics approval and consent to participate}

Because of the retrospective design of the study, formal research ethics committee approval and patients' written informed consent for publication of this manuscript and accompanying images were deemed unnecessary after consultation with the local ethics committee (Saudi German Hospital Jeddah ethics committee).

\section{Consent for publication}

Not applicable.

\section{Competing interests}

The author of this manuscript declares no relationships with any companies, whose products or services may be related to the subject matter of the article.

\section{Author details}

${ }^{1}$ Department of Diagnostic Radiology, Medical Research Institute, Alexandria University, Alexandria, Egypt. ${ }^{2}$ Cardiology Department, Faculty of Medicine, Al-Azhar University, Cairo, Egypt. ${ }^{3}$ Radiology Department, Faculty of Medicine, Ain Shams University, Cairo, Egypt.

Received: 29 July 2021 Accepted: 25 September 2021

Published online: 04 October 2021

\section{References}

1. O'Gara PT, Kushner FG, Ascheim DD et al (2013) ACCF/AHA guideline for the management of ST-elevation MI: executive summary: a report of the ACCF/AHA Task Force on Practice Guidelines. Circulation 127:529-555

2. Whalen H, Dako F, Patel P et al (2019) Role of imaging for suspected cardiac thrombus. Curr Treat Options Cardio Med 21:81

3. Bussani R, Castrichini M, Restivo L et al (2020) Cardiac tumors: diagnosis, prognosis, and treatment. Curr Cardiol Rep 22:169

4. DongJin IM, Hong SJ, Park E-A et al (2019) Guidelines for cardiovascular magnetic resonance imaging from the Korean Society of cardiovascular imaging — part 3: perfusion, delayed enhancement, and t1 - and t2 mapping. Korean J Radiol 20:1562-1582

5. Alhassan DA, Bilal WK (2020) Detection of left ventricular thrombi on cardiac magnetic resonance viability studies. JSHA 32:368-376

6. Armstrong WF, Ryan T (2010) Masses, tumors and source of embolism. In: Feigenbaum's echocardiography, 7th edn. Lippincott Williams \& Wilkins, Philadelphia, pp 711-740

7. Pinto YM, Elliott PM, Arbustini E et al (2016) Proposal for a revised definition of DCM, hypokinetic non-DCM, and its implications for clinical practice: a position statement of the ESC working group on myocardial and pericardial diseases. Eur Heart J 37:1850-1858

8. Oh JK, Park J-H, Lee J-H et al (2019) Shape and mobility of a left ventricular thrombus are predictors of thrombus resolution. KCJ 49(9):829-837

9. Smer A, Nanda N, Akdogan R et al (2020) Echocardiographic evaluation of mitral valve regurgitation. Mini-invasive Surg 4:52

10. Cerqueira MD, Weissman NJ, Dilsizian V et al (2002) Standardized myocardial segmentation and nomenclature for tomographic imaging of the heart. A statement for healthcare professionals from the Cardiac Imaging Committee of the Council on Clinical Cardiology of the AHA. Circulation 105:539-542

11. Lee E, Ibrahim E-S, Parwani P et al (2020) Practical guide to evaluating myocardial disease by cardiac MRI. AJR 214:546-556

12. Vancheri F, Longo G, Vancher S et al (2020) Coronary microvascular dysfunction. J Clin Med 9:2880

13. Osherov AB, Borovik-Raz M, Aronson D et al (2009) Incidence of early left ventricular thrombus after acute anterior wall $\mathrm{Ml}$ in the primary coronary intervention era. Am Heart J 157:1074-1080

14. Jiang YX, Jing LD, Jia YH (2015) Clinical characteristics and risk factors of left ventricular thrombus after AMI: a matched case-control Study. Chin Med J 128:2415-2419

15. Turpie AG, Robinson JG, Doyle DJ et al (1989) Comparison of high-dose with low-dose subcutaneous heparin to prevent left ventricular mural thrombosis in patients with acute transmural anterior MI. N Engl J Med 320:352

16. Vecchio C, Chiarella F, Lupi G et al (1991) Left ventricular thrombus in anterior AMI after thrombolysis. A GISSI-2 connected study. Circulation 84:512-519

17. Patel JB, Borgeson DD, Barnes ME et al (2004) Mitral regurgitation in patients with advanced systolic heart failure. J Card Fail 10:285-291

18. Delemarre BJ, Visser CA, Bot H et al (1990) Prediction of apical thrombus formation in AMI based on left ventricular spatial flow pattern. JACC 15:355

19. Garg P, Rob J, Geest VD et al (2019) Left ventricular thrombus formation in $\mathrm{Ml}$ is associated with altered left ventricular blood flow energetics. Eur Heart J Cardiovasc Imaging 20:108-117

20. Maze SS, Culture MN, Parry WE (1989) Flow characteristics in the dilated Left Ventricle with Thrombus: qualitative and quantitative Doppler analysis. JACC 13:873-881

21. Sharma ND, McCullough PA, Philbin EF et al (2000) Left ventricular thrombus and subsequent thromboembolism in patients with severe systolic dysfunction. Chest 117:314-320

22. Loh E, Sutton MSJ, Wun CC et al (1997) Ventricular dysfunction and the risk of stroke after MI. NEGM 336:251-257

23. Rengo G, Pagano G, Squizzato A et al (2013) Oral anticoagulation therapy in heart failure patients in sinus rhythm: a systematic review and meta-analysis. PLoS ONE 8:e52952

24. Roifman I, Connelly KA, Wright GA et al (2015) Echocardiography vs CMRI for the diagnosis of left ventricular thrombus: a systematic review. Can J Cardiol 31:785-791

25. Kaolawanich Y, Boonyasirinant Th (2019) Usefulness of apical area index to predict left ventricular thrombus in patients with systolic dysfunction: a novel index from CMR. BMC Cardiovasc Disord 19(15):1-8

26. Weinsaft JW, Kim HW, Shah DJ et al (2008) Detection of left ventricular thrombus by delayed-enhancement CMR: prevalence and markers in patients with systolic dysfunction. JACC 52:148-157

27. Grothues F, Smith GC, Moon JC et al (2002) Comparison of inter-study reproducibility of CMR with two-dimensional echocardiography in normal subjects and in patients with heart failure or left ventricular hypertrophy. Am J Cardiol 90:29-34 
28. Mollet NR, Dymarkowski S, Volders W et al (2002) Visualization of ventricular thrombi with contrast-enhanced magnetic resonance imaging in patients with ischemic heart disease. Circulation 106:2873-2876

29. Sürder D, Gisler V, Corti R et al (2015) Thrombus formation in the left ventricle after large Ml: assessment with CMRI. Swiss Med Wkly 145:w14122

30. Weinsaft JW, Kim J, Medicherla CB et al (2016) Echocardiographic algorithm for post-myocardial infarction LV thrombus: a gatekeeper for thrombus evaluation by delayed enhancement CMR. JACC Imaging 9:505-515

31. Broncano J, Bhalla S, Caro P et al (2021) CMRI in patients with acute chest pain. RG 41:8-31
32. Habash F, Vallurupalli S (2017) Challenges in management of left ventricular thrombus. Ther Adv Cardiovasc Dis 11:203-213

33. Weinreb J, Rodby R, Yee J et al (2021) Use of intravenous gadoliniumbased contrast media in patients with kidney disease: consensus statements from the American College of radiology and the National Kidney Foundation. Radiology 298:28-35

\section{Publisher's Note}

Springer Nature remains neutral with regard to jurisdictional claims in published maps and institutional affiliations.

\section{Submit your manuscript to a SpringerOpen ${ }^{\circ}$ journal and benefit from:}

- Convenient online submission

- Rigorous peer review

- Open access: articles freely available online

- High visibility within the field

- Retaining the copyright to your article

Submit your next manuscript at $\boldsymbol{\nabla}$ springeropen.com 